Article

\title{
Multidimensional Analysis of Time-Resolved Charged Particle Imaging Experiments
}

\author{
Vincent Loriot ${ }^{1,2,3}$ (D), Luis Bañares ${ }^{1, *(\mathbb{D})}$ and Rebeca de Nalda ${ }^{2}$ (D) \\ 1 Departamento de Química Física (Unidad Asociada I+D+i al CSIC), Facultad de Ciencias Químicas, \\ Universidad Complutense de Madrid, 28040 Madrid, Spain; vincent.loriot@univ-lyon1.fr \\ 2 Instituto de Química Física Rocasolano, CSIC, C/Serrano 119, 28006 Madrid, Spain; r.nalda@iqfr.csic.es \\ 3 Université de Lyon, Université Claude Bernard Lyon 1, CNRS, Institut Lumière Matière, \\ F-69622 Villeurbanne, France \\ * Correspondence: lbanares@ucm.es
}

Received: 13 June 2018; Accepted: 23 July 2018; Published: 26 July 2018

check for updates

\begin{abstract}
We present a tutorial to realize a multidimensional fitting procedure capable of extracting all the relevant information contained in a sequence of charged particle images acquired as a function of time in femtosecond pump-probe experiments. The images are reproduced using a 3D fitting method, which provides the velocity (or center-of-mass kinetic energy) and angular distributions contained in the images and their time evolution. A detailed example of the method is shown through the analysis of the time-resolved predissociation dynamics of $\mathrm{CH}_{3} \mathrm{I}$ on the $B$-band origin (Gitzinger et al., J. Chem. Phys. 2010, 133, 234313). We show that the multidimensional approach is essential for the analysis of complex images that contain several overlapping contributions where reduced dimensionality analyses cannot provide a reliable description of the features present in the image sequence. This methodology can be generalized to many types of multidimensional data analysis.
\end{abstract}

Keywords: femtochemistry; VMI (velocity map imaging); multidimensional analysis

\section{Introduction}

A broad range of experimental scientific fields have moved over the last few years from the study of scalar or vectorial quantities (i.e., measurement of a magnitude or a string of data as a function of a relevant variable) to the acquisition of images (i.e., 2D sets of data). The broad availability of cheap charge-coupled devices (CCD), the larger storage capabilities and the faster communication protocols have contributed to this remarkable change. In particular, in the field of Atomic and Molecular Physics, traditional methods, such as time-of-flight detection of ions or photoelectrons, are increasingly being replaced by techniques like ion or photoelectron imaging, or even ion-photoelectron coincidence imaging, for the study of photoionization, photodissociation, reaction dynamics or molecular alignment [1-8]. The acquisition of spatially-resolved data on the final position of these particles can yield a wealth of information of the process under study that was unimaginable with previous methods. In the analysis of these data, it has been common practice to analyze either integrated sections or cuts of the images to obtain 1D information that is then analyzed with standard OD or 1D fitting procedures [9-12]. In many cases, the richness of the information that can be extracted from the data is lost. Multidimensional analysis can separate certain contributions that are often the key to unravel the dynamical processes.

In this work, we present a multidimensional fitting solution dedicated to obtain the best fit to the complete set of data contained in a time sequence of a sequence of velocity map images measured in femtosecond pump-probe experiments, through parameterized functionals that describe 
radial and angular properties of the particle distribution as a function of time. As will be shown, the multidimensional fit becomes essential for instance when contributions with different time behavior are overlapping. Additionally, for those cases where the initial guesses for the parameters or functional forms of the fit are misguided (on the number or nature of the contributions to the image, on the time behavior of anisotropy, etc...), discrepancies can be detected easily through the use of the analysis of the residuals. It is important to note that the multidimensional nature of the fit allows the discrimination of the different contributions to the images, in a manner that a reduced-dimensionality analysis cannot achieve. This method has revealed its value in a number of contributions where the need for discrimination of overlapping contributions in charged particle images was crucial [13-18]. The method is analogous to the global 2D fit approach used in Stolow's group [19-22] but can easily be extended to extra dimensions such as the anisotropy, the temperature, the intensity dependence, measurements in coincidence, etc... With some modifications, the method can be equally applied to other problems, such as the detection of spectrally and spatially resolved X-rays from high-harmonic generation [23], or temporal-spectral-spatial ultrashort pulse characterization [24-26] and time-resolved time-of-flight measurements [27]. For each particular application, functional shapes and dependences have to be adapted, but the strategy presented here is of general applicability to the study of 2D or higher dimensionality data. We describe the procedure in a pedagogical way to adapt any algorithms of optimization to a multidimensional analysis.

The paper is organized as follows. In Section 2, the method to construct velocity map images and to describe their time evolution is presented, including both $3 \mathrm{D}$ and $2 \mathrm{D}$ versions. The multidimensional numerical fitting procedure is explained in Section 3, and Section 4 is dedicated to the application of the method to a case example: the femtosecond pump-probe velocity map imaging (VMI) experiment on $\mathrm{CH}_{3} \mathrm{I}$ predissociation on the $\mathrm{B}$-band. Section 5 closes the paper with the main conclusions.

\section{Construction of a Sequence of Velocity Map Images and Description of Its Time Evolution}

The first demonstration of charged-particle imaging applied to reaction dynamics was made by Chandler and Houston. In their 1987 work [1], they showed how it is possible to record, at once, the entire spatial distribution of fragments originating from a photodissociation event. In this way, within the resolution limits, it is possible to directly measure the angular and velocity distributions of the products of a chemical reaction. The technique of ion/photoelectron imaging made a giant leap a decade later with the discovery by Eppink and Parker of the technique that is known as velocity map imaging [2], where through the use of open-lens electrodes with appropriate voltages, it is possible to work in a configuration where products of a photodissociation event with the same initial velocity vector are imaged onto the same position in the detector. This implies that the observed images are in fact $2 \mathrm{D}$ projections onto the plane of the detector of a velocity distribution on a spherical surface. Inversion techniques, such as the inverse Abel transform [4,28-31], need to be implemented in order to extract the true distribution from the 2D projection. Further developments of this technique led to the discovery of slice imaging for ions [32-34], where the extraction and detection conditions are such that only the central slice of the distribution on the sphere is detected, eliminating the need for inversion procedures that invariably introduce additional noise and required the existence of cylindrical symmetry in the interaction.

A typical image acquired in this type of experiment, either raw (through slice imaging), or, equivalently, mathematically inverted (through velocity map imaging), contains, in general, a set of "contributions" in the form of isotropic or anisotropic rings, by which we mean each of the possible processes or channels associated with a given ion or photoelectron. Typically, a "channel" is characterized by ions or photoelectrons with a given velocity (or center-of-mass kinetic energy) distribution, which, on the image, can be modeled, for instance, by a radial Gaussian function of the form

$$
R(r)=\exp \left[-4 \ln 2\left(\frac{r-r_{c}}{\sigma_{c}}\right)^{2}\right],
$$


where $r_{c}$ is the radial distance from the center of the image and $\sigma_{c}$ is the full-width-at-half-maximum (FWHM) of the contribution. Figure 1a shows an example of such a contribution in the form of an isotropic ring with parameters $r_{c}=40$ and $\sigma_{c}=3$ in units of pixels (px) of the CCD camera, which are proportional to the speed of the charged species.

The angular distribution (anisotropy) of charged particles for a given radius provides additional information on the nature of the channel. In the case of inversion symmetry, the anisotropy $A$ can be written as

$$
A(\alpha) \propto 1+\sum_{n} \beta_{2 n} P_{2 n}(\cos \alpha),
$$

where $\alpha$ is the angle between the polarization axis of the electric field and the considered direction and $n$ takes maximum values of 1 and 2 for one-photon and two-photon processes, respectively. Legendre polynomials, $P_{2 n}(\cos \alpha)$, represent a complete angular basis set, which has the advantage that only few terms in Equation (2) are generally sufficient to describe the ring anisotropy. A 2D plot of this function using $\beta_{2}=2$ and $\beta_{2 n>2}=0$ is shown in Figure $1 \mathrm{~b}$.

A channel can be fully described by the product of the velocity and angular distributions, $R(r) A(\alpha)$. Figure 1c shows the result of the product of the 2D radial and angular representations shown in Figure 1a,b.
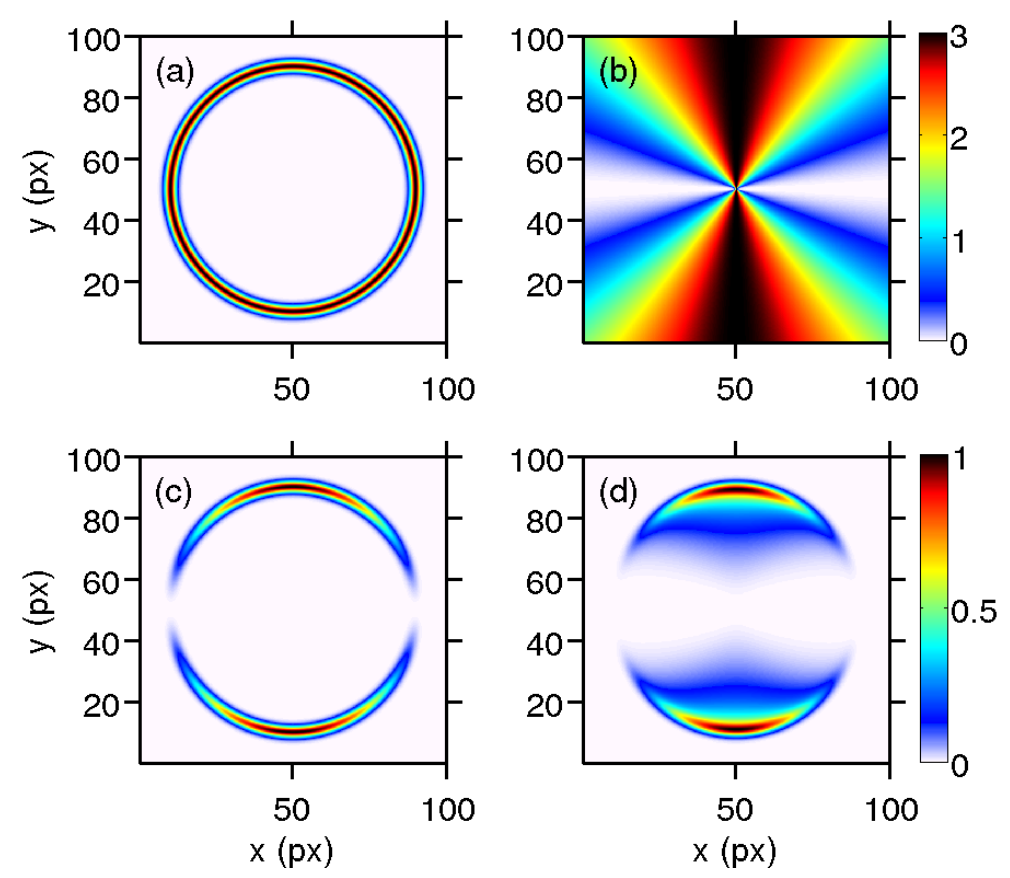

Figure 1. 2D representation of (a) the radial distribution given by Equation (1) with parameters $r_{c}=40 \mathrm{px}$ and $\sigma_{c}=3 \mathrm{px} ;(\mathbf{b})$ the angular distribution given by Equation (2) using $\beta_{2}=2$ and $\beta_{2 n>2}=0$; (c) the product of the radial and angular distributions shown in $(\mathbf{a}, \mathbf{b})$, and (d) the corresponding Abel projection of (c).

The corresponding raw velocity map image can be simulated by applying the Abel projection [4] and the result is shown in Figure 1d. This simulation of a VMI image has been obtained assuming cylindrical symmetry on the 3D distribution, for which Figure $1 \mathrm{c}$ is the central slice.

Multichannel processes lead to images $I$ with a collection of contributions $C_{i}$ like the one described above. In the case that the different contributions do not interfere with each other, the experimental signal can be described by the following sum:

$$
I=\sum_{i} a_{i} C_{i}
$$


where $a_{i}$ is the amplitude of each contribution. In the case that interferences occur, Equation (3) would include additional interference terms.

Let us now consider the particular case of a channel for which the shape of the velocity and angular distributions does not change with time. In that case, the temporal evolution $T(t)$ of the channel is represented just by an amplitude factor of the contribution that changes with time. For instance, in the case of a contribution which appears from the depopulation of an excited state (described by an exponential-type growth of the amplitude factor), the temporal behavior can be modeled as follows:

$$
T(t)=\int_{-\infty}^{\infty} \exp \left(-4 \ln 2 \frac{(t-x)^{2}}{\tau_{x c}^{2}}\right) \times\left[H\left(x-t_{0}\right) \times\left(1-\exp \left(-\frac{x-t_{0}}{\tau_{d}}\right)\right)\right] d x,
$$

or in a more symbolic form,

$$
T(t)=I_{x c}(t) \otimes\left[H\left(t-t_{0}\right) \times\left(1-e^{-\frac{t-t_{0}}{\tau_{d}}}\right)\right],
$$

where $H(t)$ is the Heaviside step function (for which $H(t<0)=0$ and $H(t>0)=1$ ) and the temporal resolution corresponds to the cross-correlation between the two laser pulses $I_{x c}(t)$, modeled by a Gaussian function with a full width at half maximum of $\tau_{x c}$, which is convoluted by the dynamics of the population of the state. The variable $t_{0}$ represents the central pump-probe delay time for which the contribution appears, and $\tau_{d}$ is the time constant of the exponential.

Therefore, the complete 3D description of a contribution $C_{i}(r, \alpha, t)$ can be written as

$$
C_{i}(r, \alpha, t)=R_{i}(r) A_{i}(\alpha) T_{i}(t)
$$

This contribution is illustrated in Figure 2.

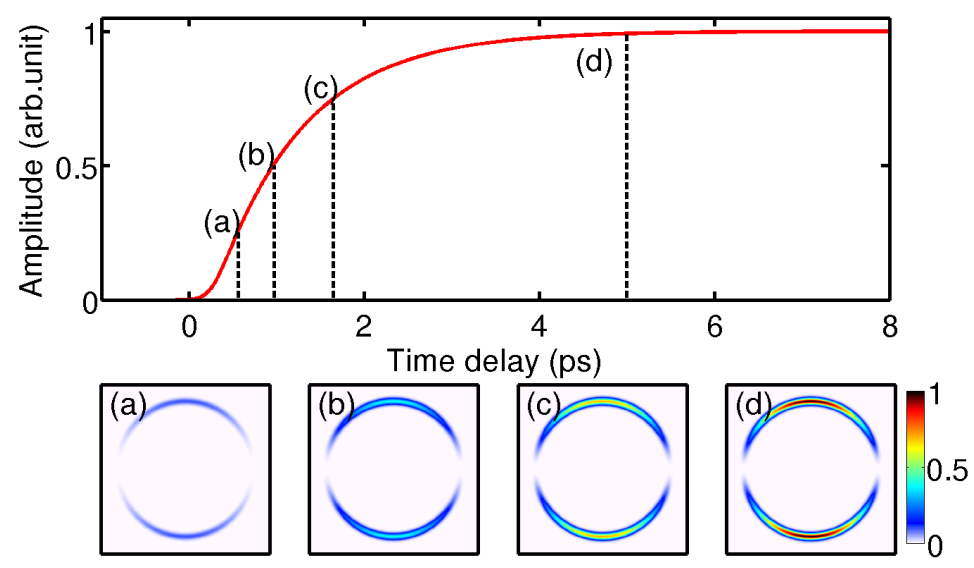

Figure 2. Time evolution of a contribution with a temporal behavior described by Equation (5) with $\tau_{d}=1 \mathrm{ps}, t_{0}=250 \mathrm{fs}$ and where $I_{x c}(t)$ is a Gaussian function with a full-width-at-half-maximum (FWHM) of $\tau_{x c}=300 \mathrm{fs}$. The images shown below correspond to the contribution depicted in Figure 1 at selected delay times. The images labelled (a-d) correspond to the time delays depicted in the transient shown in the top panel.

In the particular case described above, time appears completely decorrelated from the other dimensions. However, in a more general case, some parameters of the radial or angular distributions can change with time. For example, the anisotropy can relax, the central position of the ring can shift or its width can vary. Hence, the contribution can be written in a more general way as:

$$
C_{i}(r, \alpha, t)=R_{i}(r, t) A_{i}(\alpha, t) T_{i}(t),
$$


where now both $R$ and $A$ become functions that depend on time. The $T_{i}(t)$ function could be contained in the functional form of $R_{i}$ or $A_{i}$, but we have chosen to keep it explicitly written in Equation (7) for convenience. This formalism can be generalized in a straightforward manner to any image scan as a function of an arbitrary observable, such as wavelength or temperature, for example, thus extending the dimensionality of the problem.

In some cases, the study of the dependence of the velocity distribution with time can be sufficient to extract all the relevant information of the pump-probe VMI experiment, with no additional insight to be gained from the anisotropy. In this situation, Equation (7) can be angularly integrated and the signal originating from a given contribution becomes

$$
C_{2 D}(r, t)=R(r, t) T(t)
$$

in the case that the velocity distribution changes with time, or

$$
C_{2 D}(r, t)=R(r) T(t)
$$

if it does not.

A 2D representation (angularly integrated) of the contribution considered in Figure 2 is displayed in Figure 3a. Figure $3 b$ shows the velocity distribution (in pixels of the CCD camera) corresponding to a delay time of $5 \mathrm{ps}$. The amplitude and shape of the $2 \mathrm{D}$ and $3 \mathrm{D}$ representations can be directly compared through the angular integration

$$
C_{2 D}(r, t)=\int_{-\pi}^{\pi} r^{2} \frac{|\sin (\alpha)|}{2} C(r, \alpha, t) d \alpha
$$

In the case that the non-zero anisotropy parameters $\beta_{2 n}$ are limited to the first few orders, an alternative method to keep the 3D information consists of angularly integrating the VMI image in steps of $\Delta \theta\left(10^{\circ}\right.$ for example) providing a set of $2 \mathrm{D}$ maps as follows:

$$
C_{2 D}^{\theta_{n}}(r, t)=\int_{\theta_{n}-\Delta \theta / 2}^{\theta_{n}+\Delta \theta / 2} r^{2} \frac{|\sin (\alpha)|}{2} C(r, \alpha, t) d \alpha .
$$

In that case, the 3D fitting procedure is employed using the $n$ maps of $C_{2 D}^{\theta_{n}}(r, t)$. This last method provides the same results as the direct 3D method (Equation (7)) but tends to reduce the data size through the integration step. This can be employed in the case of slow convergence of the optimization algorithm.
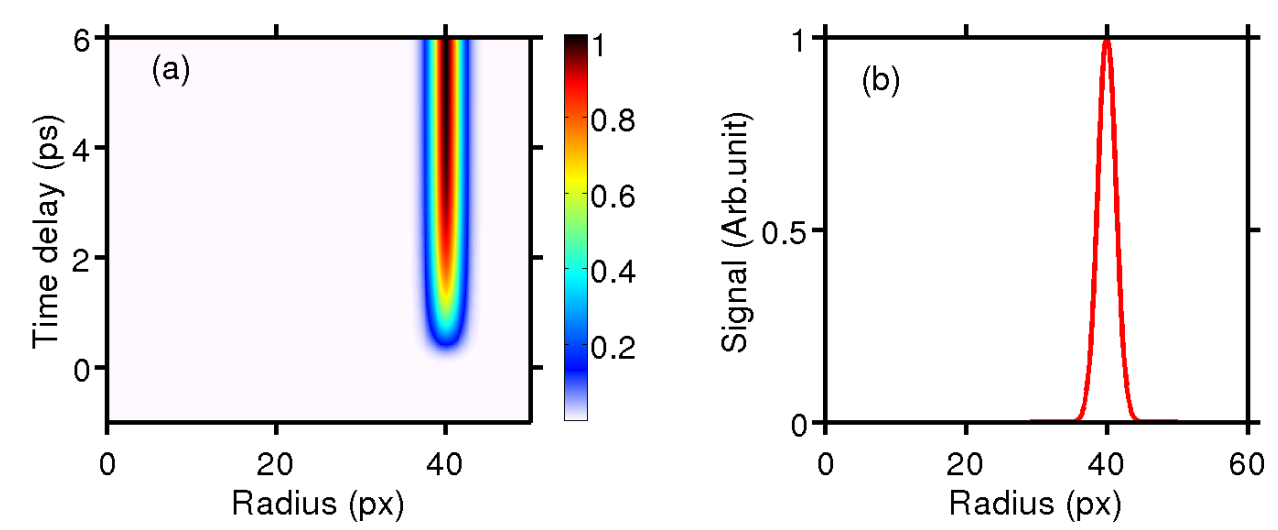

Figure 3. (a) $2 \mathrm{D}$ representation of the signal shown in Figure 2 angularly integrated as a function of radius and time; (b) velocity distribution (in pixels of the CCD camera) extracted from (a) at a fixed delay time of 5 ps. 


\section{Numerical Fitting Procedure}

The quality of a $1 \mathrm{D}$ least-squares fitting procedure is related to the possibility to produce a 1D vector, constructed from a parameterized functional form, to fit a $1 \mathrm{D}$ data vector. The most commonly available fit procedures are developed for one dimension. Typically, the routine optimizes a set of parameters, $p_{m}$, of a user-defined functional $F\left(x ; p_{m}\right)$, which minimizes the global difference between the fit and the data for all values of the $x$ variable.

A multidimensional fit can be performed using the same procedure by comparing each element of the matrix generated by the initial guess $F\left(x_{1}, x_{2}, \ldots, x_{n} ; p_{m}\right)$ with the corresponding element in the data matrix, and searching for a global minimization (in our case, the minimization algorithm employed is a Levenberg-Marquardt nonlinear regression method [35-37]). In this sense, the multidimensional fit method can be performed using the standard 1D procedure for which all the elements of the $n \mathrm{D}$ matrix are stored in a single $1 \mathrm{D}$ vector. In this approach, the multidimensional fitting procedure requires the use of invertible functions that convert an $n$-dimensional matrix into a single vector. The method of choice is to construct a $1 \mathrm{D}$ vector that contains, in queue, all the columns of the matrix and all the images of the sequence. This same conversion procedure is applied to both simulated and data matrices before and after the use of the optimisation algorithm.

A second issue concerns the choice of coordinates. Whereas the formalism of Section 2 is written in polar coordinates ( $r$, the distance from the center, and $\alpha$, the angle from the vertical axis), the experimental signal is normally recorded using a widely available 2D CCD camera with pixels that are well defined in Cartesian coordinates. We propose relating the polar and Cartesian coordinates through the advantageous complex representation:

$$
r e^{i \alpha}=i\left(x-c_{x}\right)-\left(y-c_{y}\right) d,
$$

where $\left(c_{x}, c_{y}\right)$ are the coordinates of the center of the image and $d$ is a distortion parameter of the image (different from unity in the case that the pixels are not square). In this way, $\alpha$ is defined from $-\pi$ to $\pi$ with respect to the vertical-up semi-axis, which is taken as the origin angle. In the case that the polarization axis of the laser does not match with the vertical lines of the camera but holds an angle $\delta \alpha$ with it, it can be taken into account by multiplying Equation (12) by $\exp (i \delta \alpha)$. We choose to fix $\alpha=0$ in the singular case of $r=0$ (i.e., $c_{x}$ and $c_{y}$ are integers). The images shown in Figures 1 and 2 are constructed using this conversion from polar to Cartesian coordinates.

As was mentioned before, it is common that VMI images are recorded as a function of an experimental variable of interest. Examples of this are pump-probe time delay, laser wavelength, polarization angle, temperature or sample density. This implies that a standard experiment performed in this way can produce tens or hundreds of images (for instance, $640 \times 480$ pixels, with 12-bit dynamic range). With the conditions above, a single experiment with 100 images produces $50 \mathrm{MB}$ in a stack of 3D data. Since the larger the size of the data stack, the slower the convergence of the minimization algorithm, it is important to find either symmetries or constraints that can reduce the number of data points. For example, in the case of symmetric Abel-inverted images, it is possible to work only with a square quarter of the image (corresponding to, say, $240 \times 240$ pixels). The image size can be further reduced performing an $(n \times n)$ local integration, which reduces the size by a factor $n^{2}$, although it can only be used if the spatial resolution required is not lower than $n$.

The description of a 3D functional necessarily requires a large number of parameters. The shape of each contribution along each axis and the correlations between them need to be considered. In the case of a time-resolved VMI experiment, the simplest contribution requires at least six parameters: one global amplitude, two temporal parameters (central position and time constant), two radial parameters (position and width) and one angular parameter $\left(\beta_{2}\right.$ in Equation (2)). Indeed, the number of parameters increases with the number of observed channels. However, some parameters can be common for several contributions such as the origin of time which usually corresponds to the 
temporal overlap between the pulses and the width of some of the peaks that can be related to the apparatus function.

Initial guesses for the parameters must be carefully chosen in order to reduce the convergence time. A step by step procedure is proposed as follows. In order to find reasonable initial values for the parameters and test the functionals, prospective low resolution fits are previously carried out with an $(n \times n)$ binning accompanied with a reduction of the number of images per time interval, so that the convergence time is reduced to a few minutes. The introduction of the contributions has to be performed stepwise, starting with the most intense one. A functional form for this main contribution is proposed, either empirically or from theoretical arguments, using parameters that can be estimated from visual inspection of a few images. Depending on the quality of the fit (looking at the residual), the functional can be improved. Then, the other contributions are introduced one by one using the same strategy. Finally, the final fitting is obtained by removing the binning and considering all the images.

\section{Case Example: Analysis of a Femtosecond Pump-Probe VMI Experiment}

In this section, we illustrate the strategy described in Sections 2 and 3 to the analysis of an experimental image sequence obtained in a femtosecond pump-probe VMI experiment. We take as an example the photodissociation of $\mathrm{CH}_{3} \mathrm{I}$ in the $B$-band, using a $201.2 \mathrm{~nm}$ femtosecond pump laser pulse where the appearance of the $I^{*}\left({ }^{2} P_{1 / 2}\right)$ fragment is probed by $(2+1)$ resonance enhanced multiphoton ionization (REMPI) with an ultrashort $305 \mathrm{~nm}$ laser pulse [38]. The dissociative mechanism of $\mathrm{CH}_{3} \mathrm{I}$ in the $\mathrm{B}$-band is relatively simple and consists of an electronic predissociation process [39-42]. The absorption of one pump photon at $201.2 \mathrm{~nm}$ excites the $\mathrm{CH}_{3} \mathrm{I}$ molecule from the electronic ground state into a bound Rydberg state in the ground vibrational state ( $0_{0}^{0}$ transition). This Rydberg state is crossed by a repulsive potential energy surface (PES) belonging to the $A$-band, which correlates with $\mathrm{CH}_{3}$ and $\mathrm{I}^{*}\left({ }^{2} P_{1 / 2}\right)$ fragments [43]. The initial population in the Rydberg state can be transferred into the repulsive PES with an efficiency that depends on the coupling between the two surfaces. Once the transfer is done, the dissociation of the molecule occurs in tens of femtoseconds producing $\mathrm{CH}_{3}$ and $\mathrm{I}^{*}\left({ }^{2} P_{1 / 2}\right)$. When the iodine atom is created, it can be resonantly ionized by the probe pulse using a $(2+1)$ REMPI scheme. The ionized fragments are accelerated by an external static electric field and focalized onto a microchannel plate coupled with a phosphor screen. The VMI-image (i.e., the Abel projection of the Newton sphere) is then recorded by a 12-bit Peltier cooled CCD camera. A set of images is recorded as a function of the pump-probe delay time. Each image is numerically inverted using an Abel inversion procedure [28] to retrieve the 3D velocity distribution of fragments as shown in Figure 4a,c. See Ref. [38] for further details.

As usual in VMI experiments, each image contains information about the fragment velocity and angular distributions. Typically, a number of well defined rings representing the velocity-angle distribution of given photodissociation channels can be observed in the images. In the present case, the time and anisotropy evolution of one ring is clearly observed in the sequence of images. The amplitude of the signal of the ring depends on the number of iodine ions that are detected. The width of this contribution directly depends on the rovibrational temperature of the initial molecule and that of the $\mathrm{CH}_{3}$ co-fragment, convoluted by the imaging apparatus resolution. The exact shape of the curve of the velocity distribution can be quite complicated; nevertheless, it can be reasonably approximated by Boltzmann, Gaussian or Lorentzian functions. In this experiment, the main ring can be modeled as the sum of two Gaussian functions, which represents the $\mathrm{CH}_{3}$ co-fragment in the ground vibrational state $(v=0)$ and with one quantum of vibrational excitation in the symmetric stretch mode $\left(v_{1}=1\right)$. The temporal behavior expected for the relaxation of population can be modeled by an exponential function, as shown in Equation (5). The anisotropy of a single linearly polarized photon phenomenon can be described by the second Legendre polynomial, considering $\beta_{2}$ as the only non-zero coefficient in Equation (2). The value of $\beta_{2}$ primarily depends on the angular nature of the transition, i.e., whether the transition dipole moment is parallel or perpendicular to the dissociation 
axis. For the parallel case, $\beta_{2}>0$ with a maximum value of $\beta_{2}=2$; for the perpendicular case, $\beta_{2}<0$ with an extreme value of $\beta_{2}=-1$. Both theoretical calculations and experiments for $\mathrm{CH}_{3} \mathrm{I}$ have shown that the transition from the electronic ground state to the $B$-band is of perpendicular nature. The observed final anisotropy, measured through angular distributions of the fragments, can differ from the limiting cases of parallel $\left(\beta_{2}=2\right)$ or perpendicular $\left(\beta_{2}=-1\right)$ anisotropy, mainly because rotation of the molecule prior to dissociation tends to blur the angular preference. This is especially relevant in the case of an indirect dissociation process if the time scales are similar or slower than molecular rotation.

Inspection of a few experimental images of the sequence shows that the anisotropy does evolve in this way [38], i.e., images taken at earlier delay times show a more pronounced anisotropy than those taken at later times. The present example, therefore, provides a test of the fitting procedure where one of the angular parameters is a function of time. The functional form of the loss of anisotropy with time is not known. An exponentially decreasing modulus of the $\beta_{2}$ parameter is proposed as:

$$
\beta_{2}(t)=\beta_{2_{0}}+\Delta \beta_{2} e^{-\frac{\left(t-t_{0}\right)}{\tau_{\beta_{2}}}},
$$

where $\beta_{2_{0}}$ is the asymptotic anisotropy parameter, $\beta_{2_{0}}+\Delta \beta_{2}$ is the amplitude of the anisotropy parameter at $t=t_{0}$ and $\tau_{\beta_{2}}$ is the relaxation time. Some theoretical work has been dedicated to define the final value of $\beta_{2_{0}}$ for a dissociation process that is either parallel or perpendicular, considering the dissociation time [44,45]. Those theoretical models are based on the rotation of the excited wave packet prior to dissociation. Although no predictions are available on the relaxation time, it can be estimated to be in the picosecond time scale.

The complete $3 \mathrm{D}$ function $C_{1}$ that was employed to describe the main contribution (anisotropic ring) on the $B$-band photodissociation of $\mathrm{CH}_{3} \mathrm{I}$ can be written as follows:

$$
C_{1}(r, \alpha, t)=T_{1}(t) \times A_{1}(\alpha, t) \times R_{1}(r),
$$

where

$$
\begin{gathered}
T_{1}(t)=\exp \left(-4 \ln 2\left(\frac{t^{2}}{\tau_{x c, 1}^{2}}\right)\right) \otimes\left[H\left(t-t_{1}\right) \times\left(1-e^{-\frac{\left(t-t_{1}\right)}{\tau_{d, 1}}}\right)\right], \\
A_{1}(\alpha, t)=1+\left(\beta_{2_{0}, 1}+\Delta \beta_{2,1} e^{-\frac{\left(t-t_{1}\right)}{\tau_{\beta_{2,1}}}}\right) P_{2}(\cos \alpha),
\end{gathered}
$$

and

$$
R_{1}(r)=\exp \left(-4 \ln 2\left(\frac{\left(r-r_{1, a}\right)^{2}}{\sigma_{1, a}^{2}}\right)\right)+\epsilon_{1} \exp \left(-4 \ln 2\left(\frac{\left(r-r_{1, b}\right)^{2}}{\sigma_{1, b}^{2}}\right)\right),
$$

where two Gaussian functions describe the radial contributions corresponding to $\mathrm{CH}_{3}$ fragments in the two vibrational states $v=0$ and $v_{1}=1$, and with $\epsilon$ representing the fraction of the population of $\mathrm{CH}_{3}$ in $v_{1}=1$.

Femtosecond pump-probe VMI images contain, in general, some other low intensity secondary contributions, which can arise from different mechanisms, as, for example, absorption of two pump photons, relaxation of the molecule over another state which has lower absorption probability, detection by non-resonant ionization or background gas contamination. The secondary contributions have to be considered in the fitting procedure if they are overlapped with the contribution or contributions of interest. In fact, a good reproduction of the secondary signals allows the isolation of the contribution of interest from the rest by subtraction of the previously fitted secondary signals. If these secondary contributions are neglected, then an error is generated in the fit with a magnitude corresponding to the sum of the secondary signals in the spatial overlap region.

In the present case example [38], the secondary contributions can be grouped into two classes. The first, $C_{2}$, appears in a broad range of velocities modeled by an asymmetric Gaussian function, 
i.e., with different widths $\left(\sigma_{2, a}\right.$ and $\left.\sigma_{2, b}\right)$ at each side of the maximum. It presents a $\beta_{2}$ and $\beta_{4}$ anisotropy. This contribution appears quickly at short delay times and then exponentially decreases with a constant time $\tau_{d, 2}$. Due to a possible different number of photons involved, the temporal resolution $\tau_{x c, 2}$ can be a priori different from the other contributions. The complete mathematical description can be expressed as follows:

$$
C_{2}(r, \alpha, t)=T_{2}(t) \times A_{2}(\alpha) \times R_{2}(r),
$$

with

$$
\begin{gathered}
T_{2}(t)=\exp \left(-4 \ln 2\left(\frac{t^{2}}{\tau_{x c, 2}^{2}}\right)\right) \otimes\left[H\left(t-t_{2}\right) \times\left(e^{-\frac{\left(t-t_{2}\right)}{\tau_{d, 2}}}\right)\right], \\
A_{2}(\alpha)=1+\beta_{2,2} P_{2}(\cos \alpha)+\beta_{4,2} P_{4}(\cos \alpha)
\end{gathered}
$$

and

$$
R_{2}(r)=H\left(r_{2}-r\right) \exp \left(-4 \ln 2\left(\frac{\left(r-r_{2}\right)^{2}}{\sigma_{2, a}^{2}}\right)\right)+H\left(r-r_{2}\right) \exp \left(-4 \ln 2\left(\frac{\left(r-r_{2}\right)^{2}}{\sigma_{2, b}^{2}}\right)\right) .
$$

The second minor contribution, $C_{3}$, has more localized velocities around the region of the contribution of interest (around $C_{1}$ ). Moreover, it has a temporal expression similar to $C_{1}$. Additionally, its anisotropy requires the consideration of both $\beta_{2}$ and $\beta_{4}$ constant terms. This last contribution is modeled by the following equations:

$$
C_{3}(r, \alpha, t)=T_{3}(t) \times A_{3}(\alpha) \times R_{3}(r),
$$

with

$$
\begin{gathered}
T_{3}(t)=\exp \left(-4 \ln 2\left(\frac{t^{2}}{\tau_{x c, 3}^{2}}\right)\right) \otimes\left[H\left(t-t_{3}\right) \times\left(1-e^{-\frac{\left(t-t_{3}\right)}{\tau_{d, 3}}}\right)\right], \\
A_{3}(\alpha)=1+\beta_{2,3} P_{2}(\cos \alpha)+\beta_{4,3} P_{4}(\cos \alpha),
\end{gathered}
$$

and

$$
R_{3}(r)=\exp \left(-4 \ln 2\left(\frac{\left(r-r_{3}\right)^{2}}{\sigma_{3}^{2}}\right)\right)
$$

In what follows, the specific results of the 3D fitting procedure applied to the mentioned case example are presented. Figure 4 shows two typical images, out of the sequence of 80 images recorded, fitted at short (120 fs) and asymptotic (10 ps) delay times.

In order to remove the standard 3-4 pixel region of noise produced by the Basex inversion around the central column of the images [28], the fit has been carried out four pixels away from the central axis. Those points are thus removed from the fitted and measured images shown in Figure 4. Additionally, some of the mathematical functions used in the fitting procedure present a singularity at the center of the images. In order to avoid the singularities, a 10 pixel circle was set to zero in the fitted and measured matrices. The elimination of these areas of the images does not represent any problem in the analysis, since all the relevant contributions are far away from the center.

The advantage of the 3D fitting procedure is that the anisotropy is considered. In the present case, the main contribution (ring) starts with a pronounced anisotropy (of perpendicular character), with the ion signal concentrated at the equator of the images, and later the ring evolves to become more isotropic. The anisotropy parameters of Equation (16) obtained from the fit for this main contribution are $\beta_{2_{0,1}}=-0.50 \pm 0.03, \Delta \beta_{2,1}=-0.51 \pm 0.03$ and $\tau_{\beta_{2,1}}=1.26 \pm 0.13 \mathrm{ps}$. Therefore, the fitted anisotropy at $t=t_{1}$ is $\beta_{2,1}\left(t=t_{1}\right)=-1.01 \pm 0.06$, which is characteristic of a purely perpendicular transition.

The two secondary contributions are visible in the experimental images shown in Figure 4. The first can be clearly seen at the center of the image shown in Figure 4a; the second is appreciated 
at the vicinity of the main ring in Figure $4 \mathrm{~d}$. These secondary contributions, together with the main contribution, can be distinguished in a more clear way in the velocity distributions shown in Figure 5 at two delay times.
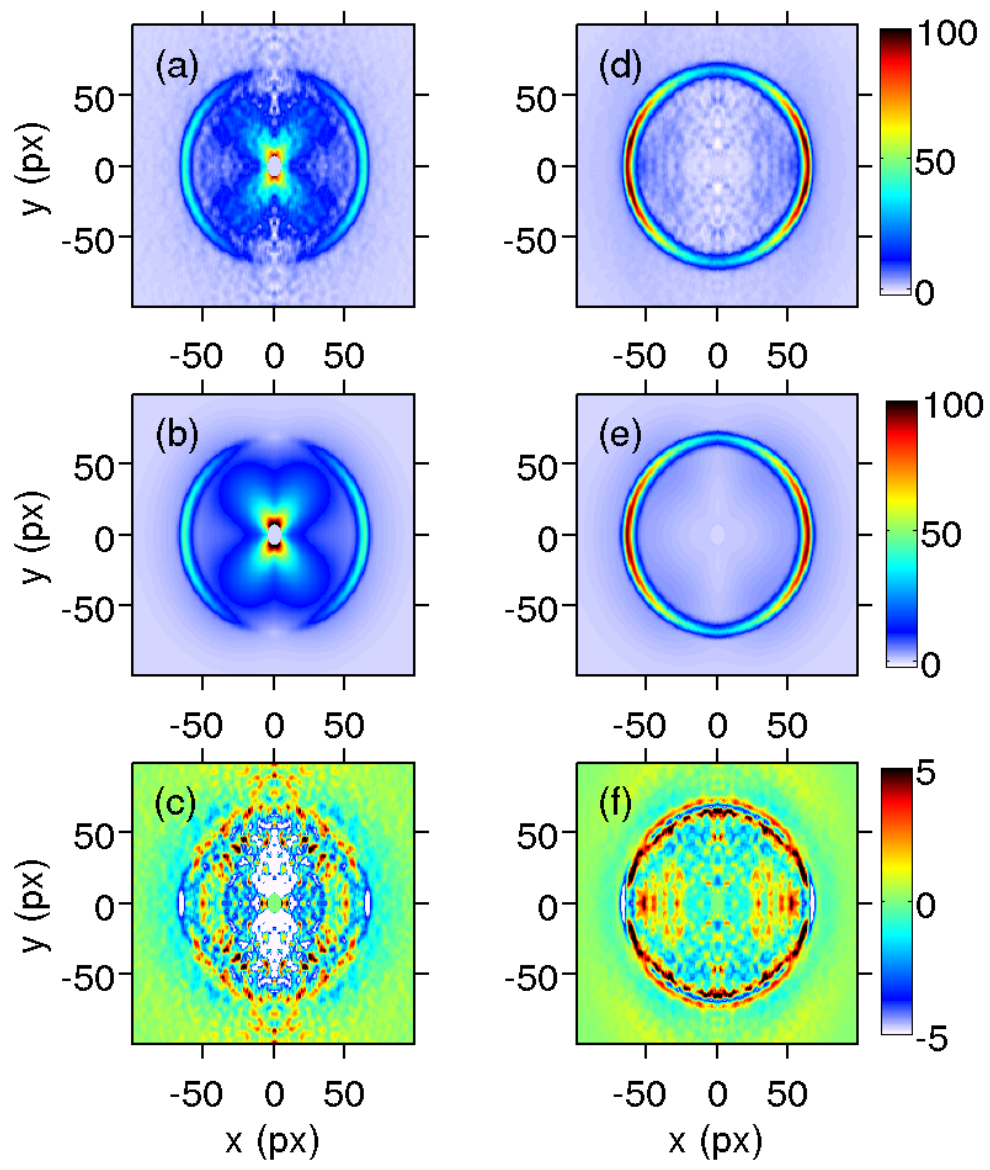

Figure 4. (a,d) experimental Abel inverted velocity map imaging (VMI) images recorded for the $I^{*}\left({ }^{2} P_{1 / 2}\right)$ fragment at 120 fs and 10 ps delay times, respectively; (b,e) corresponding fitted reconstructed images; (c,f) are the residuals corresponding to the experimental image subtracted from the fit.
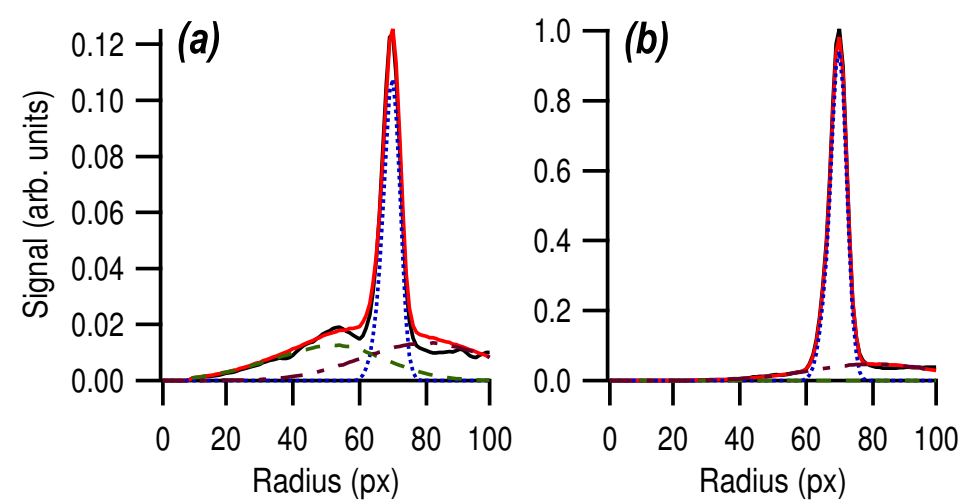

Figure 5. $\mathrm{I}^{*}\left({ }^{2} P_{1 / 2}\right)$ fragment velocity distributions (in pixels) at (a) $120 \mathrm{fs}$ and (b) 10 ps delay times. The experimental data (-), the fitted total (-) and the fitted different contributions (main $(\cdots)$ and secondary $(--$ and $\cdot-)$ ) are shown.

The time evolution of the velocity distribution can be well represented by angular integration of the images, as shown in Figure 6. 
The main contribution (ring) has been fitted in velocity (pixels) using the sum of two Gaussian functions, which have the same width $\left(\sigma_{1, a}=\sigma_{1, b}=5.27 \pm 0.04\right.$ pixel $)$ as indicated in Ref. [38]. The central position has been found to be at $r_{1, a}=69.9 \pm 0.5 \mathrm{px}$ for the main peak and at $r_{1, b}=65.7 \pm 0.5 \mathrm{px}$ for the minor shoulder with an amplitude $\epsilon=0.147 \pm 0.002$. As was indicated above, the main peak and the shoulder are assigned to dissociation channels yielding $\mathrm{CH}_{3}(v=0)$ and $\mathrm{CH}_{3}\left(\nu_{1}=1\right)$, respectively.
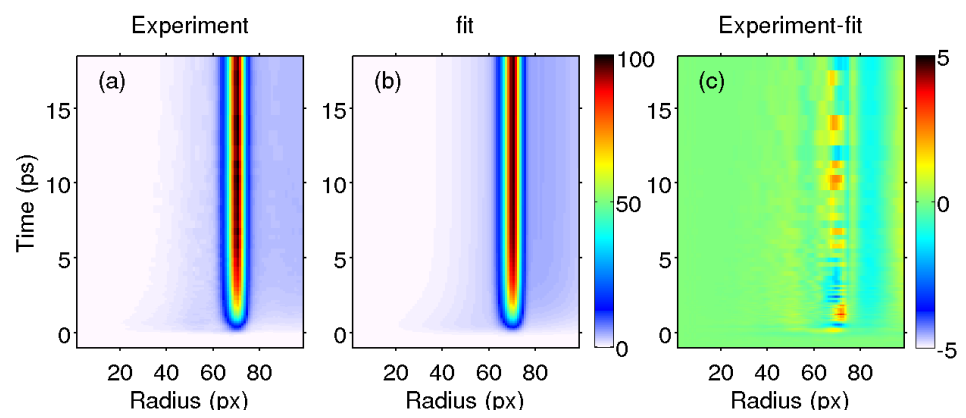

Figure 6. $2 \mathrm{D}$ representation corresponding to the angular integration of the $\mathrm{I}^{*}\left({ }^{2} P_{1 / 2}\right)$ fragment image sequence as a function of the delay time. (a) experimental measurement; (b) the corresponding fitting; and (c) the residuals corresponding to the experiment subtracted from the fit.

The temporal evolution of the secondary contribution $C_{2}$ appearing in the center of the images has been fitted using a $\tau_{x c, 2}=400 \pm 50 \mathrm{fs}$ Gaussian convolution of a $\tau_{d, 2}=1.7 \pm 0.1$ ps exponential decay. The other secondary contribution, $C_{3}$, has a time constant of $\tau_{d, 3}=1.48 \pm 0.05$ ps.

The temporal behavior of the contribution of interest $C_{1}$ isolated from the secondary contributions is shown in Figure 7 as a 1D transient. The data points shown in this figure have been obtained through subtraction of the fitted secondary contributions from the experimental measurement. Then, the resulting signal has been fully integrated angularly and radially integrated over the region of interest.

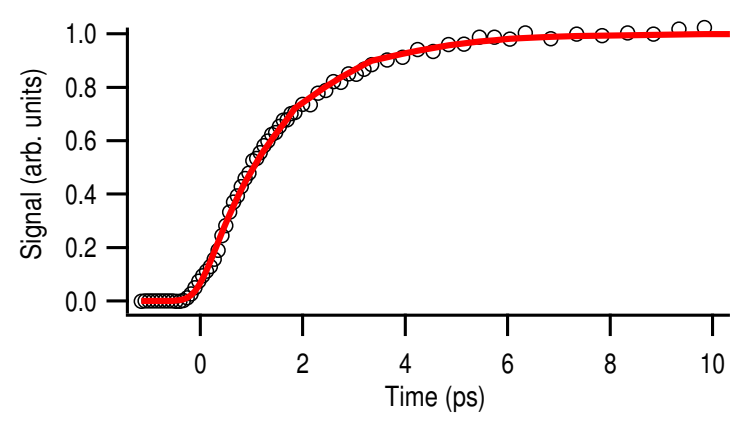

Figure 7. 1D transient for the amplitude of the main contribution as a function of time. $(O)$ experimental data and (-) fit. See text for more details.

An exponential-type growth (Equation (15)) with a time constant of $\tau_{d, 1}=1.54 \pm 0.04 \mathrm{ps}$ for this particular case has been found from the fit. Several (from 6 to around 30) independent sets of measurements across the full temporal range were acquired. Each time resolved-VMI measurement is independently fitted, keeping the same mathematical function. Each Levenberg-Marquardt fit provides fitting parameters and their corresponding standard deviations. A statistical study of the parameters that may not change with the scan, such as lifetimes of a given state, is hence performed. For such parameters, the extracted value is defined by the center of mass of the parameters found by each fitting procedure weighted by the inverse of its own standard deviation. Its respective error is 
hence the weighted dispersion of the individual values. The time constant thus obtained has been found to be $1.5 \pm 0.1 \mathrm{ps}$, as shown in Ref. [38].

The computer time required for the fit strongly depends on the chosen algorithm (Levenberg-Marquardt, genetic algorithm, ...), the possibility of parallel the calculations, the computational language, the size of the data, the number of contributions involved in the process, the number of parameters required, the quality of estimation of the initial guess and many other parameters. As an indication of the fit described in this section, considering around 30 parameters, it takes only 1-2 min with an un-paralleled CPU and a computer with an Intel core i7-2700K CPU (Santa clara, CA, USA) at 3.5 GHz with 16 Go RAM Matlab (R2013a) (Mathworks, Natick, MA, USA) based program running on Debian operating system.

\section{Conclusions}

A multidimensional fitting methodology has been developed for the full analysis of time-resolved velocity map image sequences obtained in femtosecond pump-probe photodissociation experiments. The key advantage of the method consists of its capability to distinguish the different overlapped contributions present in the set of images corresponding to different reaction channels of interest from other secondary signals arising from different mechanisms. Moreover, the contributions of interest can be cleanly isolated from the secondary signals by filtering secondary contributions from the data matrix. It must be noticed that the Abel projection can be implemented to fit recorded images directly, in which case no previous requirements on inversion symmetry are necessary.

The present multidimensional fitting procedure has been applied to the analysis of a set of images corresponding to the predissociation dynamics of $\mathrm{CH}_{3} \mathrm{I}$ in the origin of the $\mathrm{B}$-band detecting the $\mathrm{I}^{*}\left({ }^{2} P_{1 / 2}\right)$ fragments by $2+1$ REMPI. Additionally, this methodology has been applied successfully to the analysis of other dissociation channels of vibrationally excited $\mathrm{CH}_{3} \mathrm{I}$ in the $B$-band, and in the $A$-band, both for the monomer and dimer species.

This multidimensional fitting concept applied in the present case to a set of velocity map images as a function of time can be easily extended to other variables, like laser intensity, wavelength, temperature or pulse duration. It can also be adapted to other types of signal detection, like fluorescence. In addition, there is no conceptual problem to extend the fitting procedure to $n$ dimensions, the only limitation being computational time restrictions to analyze an $n$-dimensional matrix.

Author Contributions: V.L. has proposed the development of this work under the supervision of L.B. and R.N. V.L. developed the concept of the multidimensional analysis and the associated mathematical description applied to the particular case of charged particle imaging experiments and wrote the corresponding family of Matlab codes. The program has been applied to many experimental data of V.L., L.B. and R.N. V.L. wrote the initial version of the manuscript and all authors have equally contributed to the final version of the document.

Funding: This work has been supported by the Spanish Ministry of Economy and Competitiveness (Grants CTQ2015-65033-P and CTQ2016-75880-P).

Acknowledgments: This research has been carried out within the Unidad Asociada Química Física Molecular between Departamento de Química Física of Universidad Complutense de Madrid and CSIC. The facilities provided by the CLUR (Centro de Láseres Ultrarrápidos) are gratefully acknowledged.

Conflicts of Interest: The authors declare no conflict of interest.

\section{References}

1. Chandler, D.W.; Houston, P.L. Two-dimensional imaging of state-selected photodissociation products detected by multiphoton ionization. J. Chem. Phys. 1987, 87, 1445-1447. [CrossRef]

2. Eppink, A.T.J.B.; Parker, D.H. Velocity map imaging of ions and electrons using electrostatic lenses: Application in photoelectron and photofragment ion imaging of molecular oxygen. Rev. Sci. Instrum. 1997, 68, 3477-3484. [CrossRef]

3. Davies, J.A.; LeClaire, J.E.; Continetti, R.E.; Hayden, C.C. Femtosecond time-resolved photoelectron-photoion coincidence imaging studies of dissociation dynamics. J. Chem. Phys. 1999, 111, 1-4. [CrossRef] 
4. Whitaker, B.J. Imaging in Molecular Dynamics. Technology and Applications; Cambridge Univeristy Press: Cambridge, UK, 2003.

5. Ullrich, J.; Moshammer, R.; Dorn, A.; Dörner, R.; Schmidt, L.P.H.; Schmidt-Böcking, H. Recoil-ion and electron momentum spectroscopy: reaction-microscopes. Rep. Prog. Phys. 2003, 66, 1463. [CrossRef]

6. Ashfold, M.N.R.; Nahler, N.H.; Orr-Ewing, A.J.; Vieuxmaire, O.P.J.; Toomes, R.L.; Kitsopoulos, T.N.; Garcia, I.A.; Chestakov, D.A.; Wu, S.-M.; Parker, D.H. Imaging the dynamics of gas phase reactions. Phys. Chem. Chem. Phys. 2006, 8, 26-53. [CrossRef] [PubMed]

7. Hertel, I.V.; Radloff, W. Ultrafast dynamics in isolated molecules and molecular clusters. Rep. Prog. Phys. 2006, 69, 1897. [CrossRef]

8. Chichinin, A.I.; Gericke, K.-H.; Kauczok, S.; Maul, C. Imaging chemical reactions-3D velocity mapping. Int. Rev. Phys. Chem. 2009, 28, 607-680. [CrossRef]

9. De Nalda, R.; Izquierdo, J.G.; Dura, J.; Bañares, L. Femtosecond multichannel photodissociation dynamics of $\mathrm{CH}_{3} \mathrm{I}$ from the A band by velocity map imaging. J. Chem. Phys. 2007, 126, 021101. [CrossRef] [PubMed]

10. Wells, K.L.; Perriam, G.; Stavros, V.G. Time-resolved velocity map ion imaging study of $\mathrm{NH}_{3}$ photodissociation. J. Chem. Phys. 2009, 130 074308. [CrossRef] [PubMed]

11. Spesyvtsev, R.; Kirkby, O.M.; Vacher, M.; Fielding, H.H. Shedding new light on the role of the Rydberg state in the photochemistry of aniline. Phys. Chem. Chem. Phys. 2012, 14, 9942-9947. [CrossRef] [PubMed]

12. Spesyvtsev, R.; Kirkby, O.M.; Fielding, H.H. Ultrafast dynamics of aniline following 269-238 nm excitation and the role of the $\mathrm{S}_{2}\left(\pi 3 s / \pi \sigma^{*}\right)$ state. Faraday Discuss. 2012, 157 165-179. [CrossRef] [PubMed]

13. De Nalda, R.; Durá, J.; González-Vázquez, J.; Loriot, V.; Bañares, L. The primary step in the ultrafast photodissociation of the methyl iodide dimer. Phys. Chem. Chem. Phys. 2011, 13, 13295-13304. [CrossRef] [PubMed]

14. Gitzinger, G.; Corrales, M.E.; Loriot, V.; de Nalda, R.; Bañares, L. A femtosecond velocity map imaging study on B-band predissociation in $\mathrm{CH}_{3}$ I. II. The $2_{0}^{1}$ and $3_{0}^{1}$ vibronic levels. J. Chem. Phys. 2012, 136, 074303. [CrossRef] [PubMed]

15. Corrales, M.E.; Balerdi, G.; Loriot, V.; de Nalda, R.; Bañares, L. Strong field control of predissociation dynamics. Faraday Discuss. 2013, 163, 447-460. [CrossRef] [PubMed]

16. Corrales, M.E.; Loriot, V.; Balerdi, G.; González-Vázquez, J.; de Nalda, R.; Bañares, L.; Zewail, A.H. Structural dynamics effects on the ultrafast chemical bond cleavage of a photodissociation reaction. Phys. Chem. Chem. Phys. 2014, 16, 8812-8818 . [CrossRef] [PubMed]

17. Balerdi, G.; Woodhouse, J.; Zanchet, A.; de Nalda, R.; Senent, M.L.; García-Vela, A.; Bañares, L. Femtosecond predissociation dynamics of the methyl radical from the $3 p_{z}$ Rydberg state. Phys. Chem. Chem. Phys. 2016, 18, 110-118. [CrossRef] [PubMed]

18. Kirkby, O.M.; Sala, M.; Balerdi, G.; de Nalda, R.; Bañares, L.; Guérin, S.; Kaltsoyannis, N.; Fielding, H. Comparing the electronic relaxation dynamics of aniline and $\mathrm{d}_{7}$-aniline following excitation at 272-238 $\mathrm{nm}$. Phys. Chem. Chem. Phys. 2015, 17, 16270-16276. [CrossRef] [PubMed]

19. Townsend, D.; Satzger, H.; Ejdrup, T.; Lee, A.M.D.; Stapelfeldt, H.; Stolow, A. ${ }^{1} B_{2}\left({ }^{1} \Sigma_{u}^{+}\right)$excited state decay dynamics in $\mathrm{CS}_{2}$. J. Chem. Phys. 2006, 125, 234302 [CrossRef] [PubMed]

20. Ullrich, S.; Schultz, T.; Zgierski, M.Z.; Stolow, A. Electronic relaxation dynamics in DNA and RNA bases studied by time-resolved photoelectron spectroscopy. Phys. Chem. Chem. Phys. 2004, 6, 2796-2801. [CrossRef]

21. Boguslavskiy, A.E.; Schalk, O.; Gador, N.; Glover, W.J.; Mori, T.; Schultz, T.; Schuurman, M.S.; Martínez, T.J.; Stolow, A. Excited state non-adiabatic dynamics of the smallest polyene, trans 1,3-butadiene. I. Time-resolved photoelectron-photoion coincidence spectroscopy. J. Chem. Phys. 2018, 148, 164302. [CrossRef] [PubMed]

22. Schalk, O.; Boguslavskiy, A.E.; Stolow, A. Substituent Effects on Dynamics at Conical Intersections: Cyclopentadienes. J. Phys. Chem. A 2010, 114, 4058-4064. [CrossRef] [PubMed]

23. Loriot, V.; Marciniak, A.; Quintard, L.; Despré, V.; Schindler, B.; Compagnon, I.; Concina, B.; Celep, G.; Bordas, C.; Catoire, F.; et al. Resolving XUV induced femtosecond and attosecond dynamics in polyatomic molecules with a compact attosecond beamline. J. Phys. Conf. Ser. 2015, 635, 012006. [CrossRef]

24. Loriot, V.; Marciniak, A.; Karras, G.; Schindler, B.; Renois-Predelus, G.; Compagnon, I.; Concina, B.; Brédy, R.; Celep, G.; Bordas, C.; et al. Angularly resolved RABBITT using a second harmonic pulse. J. Opt. 2017, 19, 114003. [CrossRef]

25. Loriot, V.; Gitzinger, G.; Forget, N. Self-referenced characterization of femtosecond laser pulses by chirp scan. Opt. Express 2013, 21, 24879-24893. [CrossRef] [PubMed] 
26. Loriot, V.; Mendoza-Yero, O.; Pérez-Vizcaíno, J.; Mínguez-Vega, G.; de Nalda, R.; Bañares, L.; Lancis, J. Fresnel phase retrieval method using an annular lens array on an SLM. Appl. Phys. B 2014, 117, 67-73. [CrossRef]

27. Marciniak, A.; Despré, V.; Barillot, T.; Rouzée, A.; Galbraith, M.C.E.; Klei, J.; Yang, C.-H.; Smeenk, C.T.L.; Loriot, V.; Reddy, S.N.; et al. XUV excitation followed by ultrafast non-adiabatic relaxation in PAH molecules as a femto-astrochemistry experiment. Nat. Commun. 2015, 6, 7909. [CrossRef] [PubMed]

28. Dribinski, V.; Ossadtchi, A.; Mandelshtam, V.A.; Reisler, H. Reconstruction of Abel-transformable images: The Gaussian basis-set expansion Abel transform method. Rev. Sci. Instrum. 2002, 73, 2634-2642. [CrossRef]

29. Garcia, G.A.; Nahon, L.; Powis, I. Two-dimensional charged particle image inversion using a polar basis function expansion. Rev. Sci. Instrum. 2004, 75, 4989-4996. [CrossRef]

30. Bordas, C.; Paulig, F.; Helm, H.; Huestis, D.L. Photoelectron imaging spectrometry: Principle and inversion method. Rev. Sci. Instrum. 1996, 67, 2257-2268. [CrossRef]

31. Vrakking, M.J.J. An iterative procedure for the inversion of two-dimensional ion/photoelectron imaging experiments. Rev. Sci. Instrum. 2001, 72, 4084-4089. [CrossRef]

32. Gebhardt, C.R.; Rakitzis, T.P.; Samartzis, P.C.; Ladopoulos, V.; Kitsopoulos, T.N. Slice imaging: A new approach to ion imaging and velocity mapping. Rev. Sci. Instrum. 2001, 72, 3848-3853. [CrossRef]

33. Townsend, D.; Minitti, M.P.; Suits, A.G. Direct current slice imaging. Rev. Sci. Instrum. 2003, 74, $2530-2539$. [CrossRef]

34. Lin, J.J.; Zhou, J.; Shiu, W.; Liu, K. Application of time-sliced ion velocity imaging to crossed molecular beam experiments. Rev. Sci. Instrum. 2003, 74, 2495-2500. [CrossRef]

35. Marquardt, D.W. An Algorithm for Least-Squares Estimation of Nonlinear Parameters. SIAM J. Appl. Math. 1963, 11, 431-441. [CrossRef]

36. Bard, Y. Nonlinear Parameter Estimation; Academic Press: New York, NY, USA, 1974.

37. Draper, N.R.; Smith, H. Applied Regression Analysis (Wiley Series in Probability and Statistics); John Wiley \& Sons Inc.: New York, NY, USA, 1981.

38. Gitzinger, G.; Corrales, M.E.; Loriot, V.; Amaral, G.A.; de Nalda, R.; Bañares, L. A femtosecond velocity map imaging study on B-band predissociation in $\mathrm{CH}_{3}$ I. I. The band origin. J. Chem. Phys. 2010, 132, 234313. [CrossRef] [PubMed]

39. Donaldson, D.J.; Vaida, V.; Naaman, R. Ultraviolet absorption spectroscopy of dissociating molecules: Effects of cluster formation on the photodissociation of $\mathrm{CH}_{3}$ I. J. Chem. Phys. 1987, 87, 2522. [CrossRef]

40. Wang, P.G.; Ziegler, L.D. Mode-specific subpicosecond photodissociation dynamics of the methyl iodide $B$ state. J. Chem. Phys. 1991, 95, 288-296. [CrossRef]

41. Syage, J.A. Predissociation lifetimes of the $\tilde{B}$ and $\tilde{C}$ Rydberg states of $\mathrm{CH}_{3}$ I. Chem. Phys. Lett. 1993, 212, 124-128. [CrossRef]

42. Baronavski, A.P.; Owrutsky, J.C.; Vibronic dependence of the $\tilde{\mathrm{B}}$ state lifetimes of $\mathrm{CH}_{3} \mathrm{I}$ and $\mathrm{CD}_{3} \mathrm{I}$ using femtosecond photoionization spectroscopy. J. Chem. Phys. 1998, 108, 3445-3452. [CrossRef]

43. Alekseyev, A.B.; Liebermann, H.-P.; Buenker, R.J.; Yurchenko, S.N. An ab initio study of the $\mathrm{CH}_{3} \mathrm{I}$ photodissociation. I. Potential energy surfaces. J. Chem. Phys. 2007, 126, 234102. [CrossRef] [PubMed]

44. Jonah, C. Effect of Rotation and Thermal Velocity on the Anisotropy in Photodissociation Spectroscopy. J. Chem. Phys. 1971, 55, 1915-1922. [CrossRef]

45. Waldeck, J.R.; Shapiro, M.; Bersohn, R. Theory of transient anisotropy in molecular photodissociation. J. Chem. Phys. 1993, 99, 5924-5935. [CrossRef]

(c) 2018 by the authors. Licensee MDPI, Basel, Switzerland. This article is an open access article distributed under the terms and conditions of the Creative Commons Attribution (CC BY) license (http://creativecommons.org/licenses/by/4.0/). 